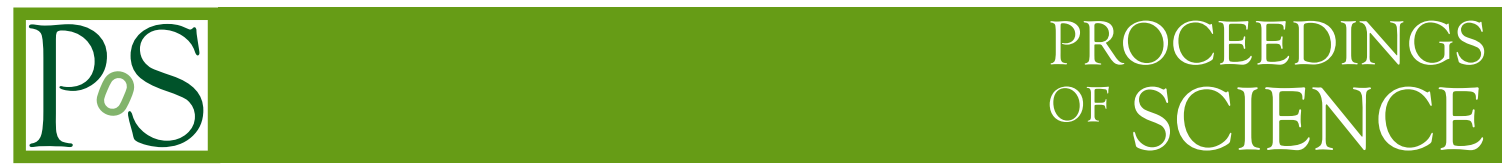

\title{
Measurements of photon- and Z-tagged jet quenching by
} ATLAS

\author{
Jeff Ouellette, on behalf of the ATLAS Collaboration ${ }^{a, *}$ \\ ${ }^{a}$ University of Colorado Boulder, \\ Boulder, CO, 80302 \\ E-mail: jeff.ouellette@cern.ch
}

The latest measurements of jet quenching by ATLAS with photon- and Z-tagged events are presented, as described during a parallel talk in the Hard Probes 2020 conference. Emphasis is on the newest measurement using $Z$-tagged events.

HardProbes 2020

1-6 June 2020

Austin, Texas

${ }^{*}$ Speaker 


\section{Introduction}

Studies of jets produced in events tagged by an electroweak boson have proven a useful way to study bulk properties of the Quark-Gluon Plasma created in heavy ion collisions. Due to the colorless nature of the electroweak bosons, such events provide an experimental handle on the initial $p_{\mathrm{T}}$ of the hard-scattered parton before parton-medium interactions begin (i.e. at the very beginning of the post-collision evolution). This provides both an initial-state snapshot of the parton as well as a final-state measurement of the modified shower, which helps understand the nature of partonmedium interactions. Previous measurements at the LHC by ATLAS have used photon-tagged events to study partonic energy loss in this manner, including studies of the photon-jet momentum balance $x_{\mathrm{j} \gamma}$ [1] and the fragmentation pattern of jets in photon-tagged events [2] with the 2015 collision data.

New, high luminosity $\mathrm{Pb}+\mathrm{Pb}$ data recorded in 2018 , at $5.02 \mathrm{TeV}$ per nucleon pair, enable new measurements of jet quenching in previously inaccessible channels - namely in events tagged by a $Z$ boson. These proceedings summarize a preliminary measurement of hadron yields in $Z$ bosontagged events in $p p$ and $\mathrm{Pb}+\mathrm{Pb}$ collisions [3], which is now finalized in Ref. [4]. This measurement uses the yield of charged particles opposite the $Z$ boson in the transverse plane as a proxy for the parton emerging from the hard scattering. No reconstructed jet is required in this measurement to investigate partonic energy loss without bias on the final state. Charged particles are selected with transverse momenta $p_{\mathrm{T}}^{\mathrm{ch}}>1 \mathrm{GeV}$ and required to be opposite the $Z$ boson in the transverse plane with $\Delta \phi>3 \pi / 4$. The yields of these charged particles are normalized per $Z$ boson and compared between $\mathrm{Pb}+\mathrm{Pb}$ and $p p$ by taking the ratio $I_{\mathrm{AA}}$. Both the yields and ratios are reported as a function of $p_{\mathrm{T}}^{\text {ch }}$ and the hadron-to-boson $p_{\mathrm{T}}$ ratio $x_{\mathrm{hZ}} \equiv p_{\mathrm{T}}^{\mathrm{ch}} / p_{\mathrm{T}}^{\mathrm{Z}}$. The measurement is performed for two different $p_{\mathrm{T}}^{\mathrm{Z}}$ selections to control for the initial hard-scattered parton $p_{\mathrm{T}}$ and for different $\mathrm{Pb}+\mathrm{Pb}$ centralities to control for the size of the nuclear overlap region.

\section{Analysis}

The ATLAS detector [5] is a large, general purpose particle detector with tracking, calorimeter, and muon spectrometer systems. $260 \mathrm{pb}^{-1}$ of $p p$ and $1.7 \mathrm{nb}^{-1}$ of $\mathrm{Pb}+\mathrm{Pb}$ collision data were used in this measurement, taken in 2017 and 2018 respectively. $Z$ boson events were recorded during data taking with single lepton triggers using the ATLAS high level trigger system.

Similar to previous measurements of $Z$ boson production [6,7], events with a $Z$ boson are tagged via their decays to opposite-sign electron or muon pairs. Candidate leptons are required to match to a trigger, have a $p_{\mathrm{T}}>20 \mathrm{GeV}$ and meet a set of identification criteria. $Z$ bosons are then reconstructed from lepton pairs with an invariant mass in the range $76<m_{Z}<106 \mathrm{GeV}$. Events are grouped based on the $Z$ boson $p_{\mathrm{T}}$, with two selections: $30<p_{\mathrm{T}}^{\mathrm{Z}}<60 \mathrm{GeV}$ and $p_{\mathrm{T}}^{\mathrm{Z}}>60 \mathrm{GeV}$. $1,300(9,000) Z \rightarrow e e$ and $1,500(11,000) Z \rightarrow \mu \mu$ events were selected with this method in $\mathrm{Pb}+\mathrm{Pb}$ $(p p)$ collisions. The total transverse energy produced at forward rapidity, $3.2<|\eta|<4.9$, is used to determine centrality percentiles. For this study, events were divided into three categories: $0-10 \%$ (“central events"), 10-30\%, and 30-80\% ("peripheral events"). Typically, central events correspond to collisions with larger nuclear overlap size and produce a larger QGP medium per collision. 

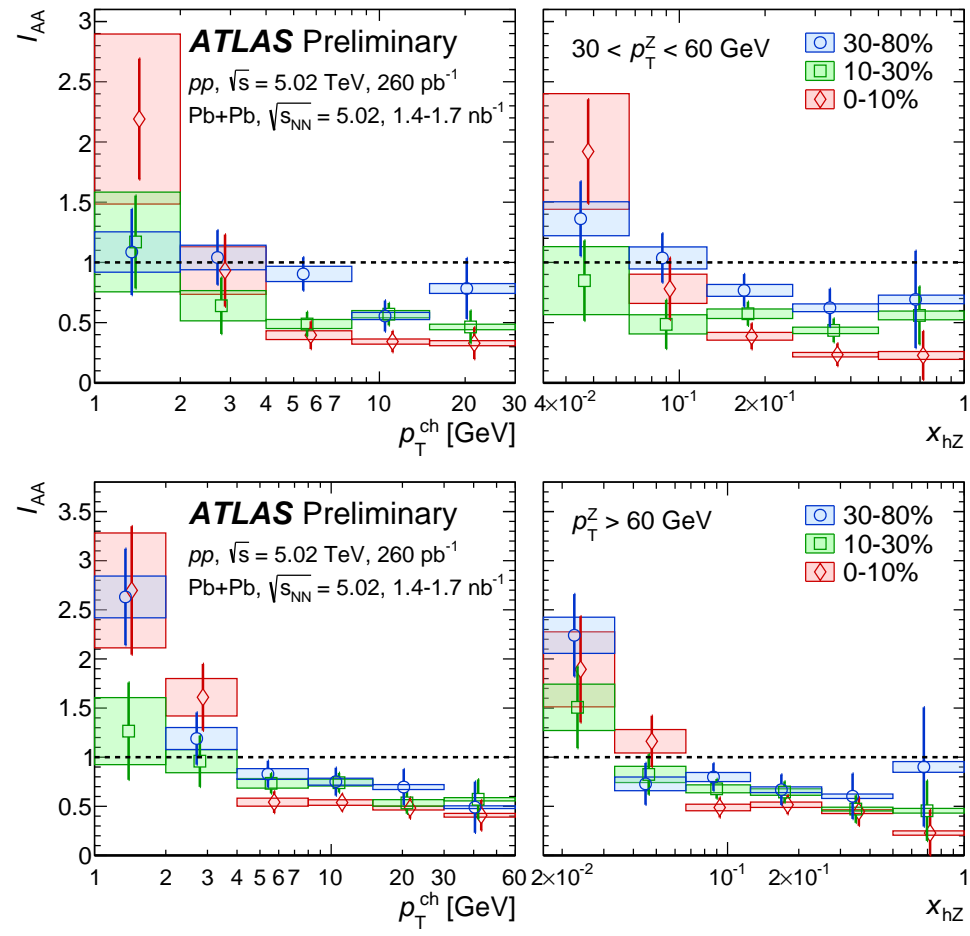

Figure 1: Ratio $I_{\mathrm{AA}}$ of the hadron yield opposite to a $Z$ boson, normalized per- $Z$, between $\mathrm{Pb}+\mathrm{Pb}$ and $p p$ collisions. Events are separated by $\mathrm{Pb}+\mathrm{Pb}$ centrality $(30-80 \%$ in blue circles, $10-30 \%$ in green squares, and $0-10 \%$ in red diamonds) and by two different $Z$ boson $p_{\mathrm{T}}\left(p_{\mathrm{T}}^{\mathrm{Z}}\right)$ selections: $30<p_{\mathrm{T}}^{\mathrm{Z}}<60 \mathrm{GeV}$ (top) and $p_{\mathrm{T}}^{\mathrm{Z}}>60 \mathrm{GeV}$ (bottom). Points are shown as a function of $p_{\mathrm{T}}^{\mathrm{ch}}$ (left) and as a function of the momentum balance $x_{\mathrm{hZ}} \equiv p_{\mathrm{T}}^{\mathrm{ch}} / p_{\mathrm{T}}^{\mathrm{Z}}$ (right).

Charged particle tracks are reconstructed using the inner detector subsystems, with the same procedure for track reconstruction as used in previous studies [2, 8]. Charged particles are required to have transverse momentum $p_{\mathrm{T}}^{\mathrm{ch}}>1 \mathrm{GeV}$ and pseudorapidity $|\eta|<2.5$. A set of quality requirements such as a minimum number of silicon hits and vertex matching criteria were applied to reject potential fake tracks. The underlying event (UE), which is generally large at low $p_{\mathrm{T}}^{\text {ch }} \lesssim 2-$ $3 \mathrm{GeV}$ and in more central $\mathrm{Pb}+\mathrm{Pb}$ collisions, is subtracted off statistically with a mixed event procedure. $Z$ bosons in both $p p$ and $\mathrm{Pb}+\mathrm{Pb}$ were mixed with minimum bias events recorded during the same data taking periods, with $\mathrm{Pb}+\mathrm{Pb}$ mixed events also required to closely match $Z$ events in centrality. The number of UE particles per signal particle was roughly 100 -to- 1 at low $p_{\mathrm{T}}$ in central events.

Both tracks and $Z$ events were weighted to account for finite triggering and reconstruction effects. Trigger efficiencies were measured per-lepton in data [9], whereas reconstruction and selection efficiencies were determined in Monte Carlo simulations by matching leptons at the truth and reconstructed levels. Possible effects from $p_{\mathrm{T}}$ migration of $Z$ bosons were accounted for by small bin-by-bin correction factors (derived in simulation) which were applied to the UE subtracted charged particle yields. Per-track corrections were also applied to account for both finite charged particle reconstruction efficiency and imperfect track purity. Several sources of systematic uncertainty were considered, including those associated with charged particle reconstruction, lepton 


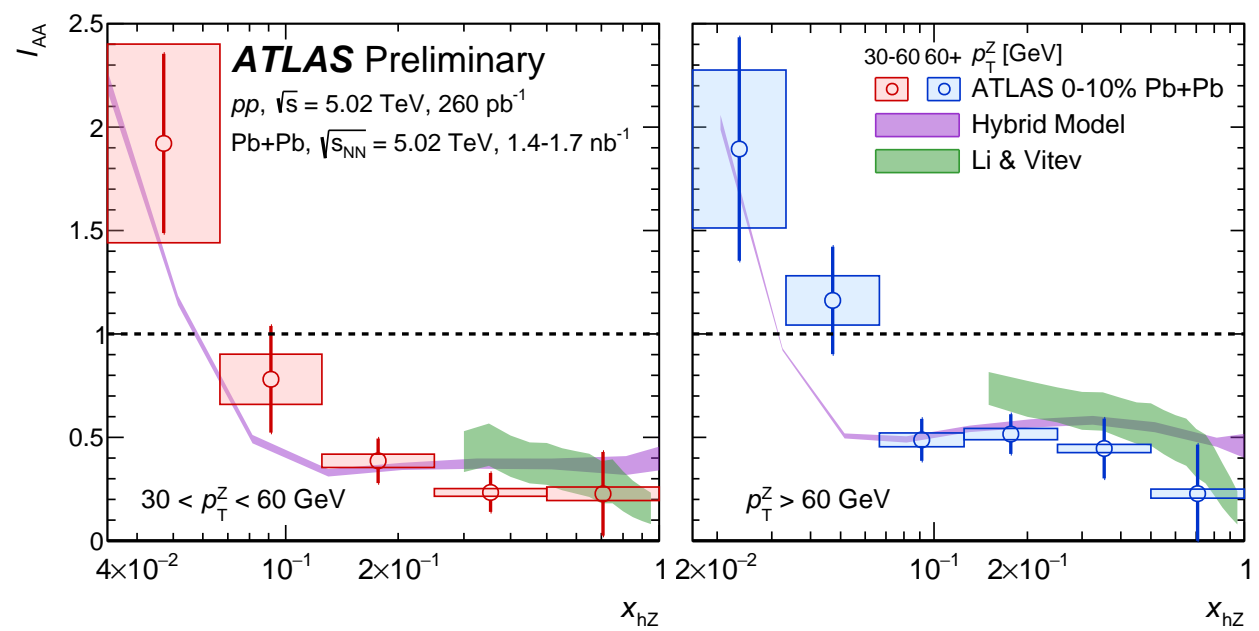

Figure 2: $I_{\mathrm{AA}}$ in $0-10 \%$ central $\mathrm{Pb}+\mathrm{Pb}$ events is compared between two different jet quenching models (see text) as a function of the momentum balance $x_{\mathrm{hZ}} \equiv p_{\mathrm{T}}^{\mathrm{ch}} / p_{\mathrm{T}}^{\mathrm{Z}}$. Events with $30<p_{\mathrm{T}}^{\mathrm{Z}}<60 \mathrm{GeV}$ and $p_{\mathrm{T}}^{\mathrm{Z}}>60 \mathrm{GeV}$ are shown on the left and right, respectively.

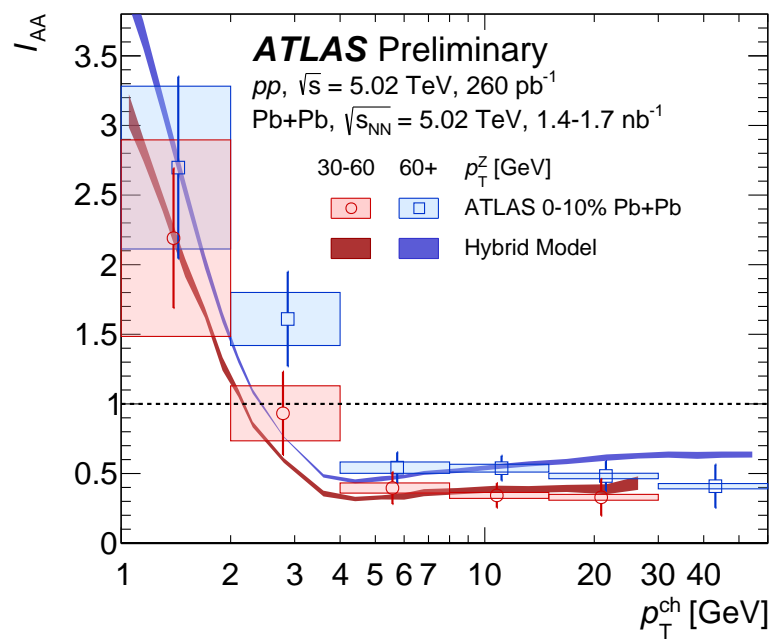

Figure 3: $I_{\mathrm{AA}}$ in $0-10 \%$ central $\mathrm{Pb}+\mathrm{Pb}$ events is compared with predictions in the hybrid strong/weak coupling model (see text) as a function of $p_{\mathrm{T}}^{\text {ch }}$ for events with $30<p_{\mathrm{T}}^{\mathrm{Z}}<60 \mathrm{GeV}$ (red) and $p_{\mathrm{T}}^{\mathrm{Z}}>60 \mathrm{GeV}$ (blue).

energy scale uncertainties, and uncertainties on the UE, the latter of which were dominant.

\section{Results}

Figure 1 presents the ratio $I_{\mathrm{AA}}$ between per- $Z$ charged particle yields in $\mathrm{Pb}+\mathrm{Pb}$ and $p p$ events. At low values of $p_{\mathrm{T}}^{\mathrm{ch}} \lesssim 3-4 \mathrm{GeV}$ there is evidence for an enhancement of charged particles in $\mathrm{Pb}+\mathrm{Pb}$ collisions, whereas above this range there is a strong suppression. The degree of suppression is found to be systematically greater in central compared to peripheral $\mathrm{Pb}+\mathrm{Pb}$ collisions, reflecting expectation that hard-scattered partons will redistribute their energy to the QGP. 
Different jet quenching models reflect this trend but the exact details differ model-to-model. In Fig. $2 I_{\mathrm{AA}}$ is compared to two different jet quenching theory models. The first is a hybrid strong/weak coupling model, which uses the gauge/gravity duality to model the dynamics of partonic energy loss [10], and is shown in purple. This calculation includes particles originating in the wake of the parton as it traverses the medium. In green, a calcuation within the framework of soft collinear effective theory $\left(\mathrm{SCET}_{\mathrm{G}}\right.$, labelled "Li \& Vitev") models parton-medium interaction with Glauber gluon interactions [11, 12], with systematic uncertainty on the parton-medium coupling $g=2.0 \pm 0.2$ shown by shaded bands. Both models reproduce the suppression of high- $x_{\mathrm{h} Z}$ charged particles observed in data, and the hybrid model produces a similar level of particle enhancement that is observed in data, rising to $I_{\mathrm{AA}} \sim 2$ around $x_{\mathrm{hZ}} \sim 0.02-0.04$. In the case of the $\mathrm{SCET}_{\mathrm{G}}$, the $x_{\mathrm{h} Z}$ dependence of the suppression matches the data well, although the overall magnitude is shifted to larger values, suggesting that a slight increase in the coupling might match the data better. For the hybrid model, the value of $x_{\mathrm{hZ}}$ at which $I_{\mathrm{AA}}$ is unity seems slightly too low compared to data. This is reflected in Fig. 3, where the two $p_{\mathrm{T}}^{\mathrm{Z}}$ selections are compared with the hybrid model as a function of $p_{\mathrm{T}}^{\mathrm{ch}}$.

\section{Conclusions}

These proceedings present the first measurement of the modification to per- $Z$ charged particle yields in $\mathrm{Pb}+\mathrm{Pb}$ collisions. The enhancement-suppression pattern exhibits a centrality and $p_{\mathrm{T}}^{\mathrm{ch}}\left(x_{\mathrm{hZ}}\right)$ dependence consistent with the parton shower being modified through its interactions with the QGP. This pattern is reproduced by theoretical predictions of $Z$-tagged charged hadron yields.

\section{References}

[1] ATLAS Collaboration, Measurement of photon-jet transverse momentum correlations in 5.02 $\mathrm{TeV} \mathrm{Pb}+\mathrm{Pb}$ and $p p$ collisions with ATLAS. Phys. Lett. B 789, 167 (2019).

[2] ATLAS Collaboration, Comparison of Fragmentation Functions for Jets Dominated by Light Quarks and Gluons from $p p$ and Pb+Pb Collisions in ATLAS. Phys. Rev. Lett. 123, 042001 (2019).

[3] ATLAS Collaboration, Measurement of Z-tagged charged-particle yields in $5.02 \mathrm{TeV} \mathrm{Pb+Pb}$ and pp collisions with the ATLAS detector. ATLAS-CONF-2019-052 (2019), Available at https://cds.cern.ch/record/2698294.

[4] ATLAS Collaboration, Medium-induced modification of Z-tagged charged particle yields in $\mathrm{Pb}+\mathrm{Pb}$ collisions at 5.02 TeV with the ATLAS detector. (2020), arXiv:2008.09811 [nucl-ex].

[5] ATLAS Collaboration, The ATLAS Experiment at the CERN Large Hadron Collider. JINST 3 (2008) S08003.

[6] ATLAS Collaboration, Measurements of $W$ and $Z$ boson production in pp collisions at $\sqrt{s}=$ 5.02 TeV with the ATLAS detector. Eur. Phys. J. C 79, 128 (2019). 
[7] ATLAS Collaboration, $Z$ boson production in $P b+P b$ collisions at $\sqrt{s_{\mathrm{NN}}}=5.02$ TeV measured by the ATLAS experiment. Phys. Lett. B 802, 135262 (2020).

[8] ATLAS Collaboration, Measurement of angular and momentum distributions of charged particles within and around jets in $\mathrm{Pb}+\mathrm{Pb}$ and $\mathrm{pp}$ collisions at $\sqrt{s_{\mathrm{NN}}}=5.02 \mathrm{TeV}$ with the ATLAS detector. Phys. Rev. C 100, 064901 (2019).

[9] ATLAS Collaboration, Performance of electron and photon triggers in ATLAS during LHC Run 2. Eur. Phys. J. C 80, 47 (2020).

[10] J. Casalderrey-Solana et al., A hybrid strong/weak coupling approach to jet quenching. JHEP 10, 019 (2014).

[11] Y.-T. Chien and I. Vitev, Towards the understanding of jet shapes and cross sections in heavy ion collisions using soft-collinear effective theory. JHEP 05, 023 (2016).

[12] H. T. Li and I. Vitev, Jet charge modification in finite QCD matter. Phys. Rev. D 101, 076020 (2020). 